\title{
Chest Wall Deformity in the Radiation Oncology Clinic
}

\author{
JOHN M. STAHL ${ }^{1}$, JULIAN C. HONG ${ }^{2}$, NATANIEL H. LESTER-COLL ${ }^{1}$, BENJAMIN H. KANN ${ }^{1}$, \\ LYNN D. WILSON ${ }^{1}$, SUSAN A. HIGGINS ${ }^{1}$ and SUZANNE B. EVANS ${ }^{1}$ \\ ${ }^{I}$ Department of Therapeutic Radiology, Yale University School of Medicine, New Haven, CT, U.S.A.; \\ ${ }^{2}$ Department of Radiation Oncology, Duke University School of Medicine, Durham, NC, U.S.A.
}

\begin{abstract}
Background/Aim: To determine the incidence of pectus excavatum $(P E)$ and define anatomical chest wall metrics predictive of elevated mean heart dose (MHD) in patients with left-sided breast cancer receiving adjuvant radiotherapy. Patients and Methods: We reviewed 273 consecutively simulated patients from 1/2013-12/2014. Physical examination identified patients with clinically identifiable PE. Characteristics were compared between patients with and those without PE. Predictors of MHD were evaluated using linear regression. Results: The average MHD for the entire cohort was 172 cGy. Of 273 patients, 10 (3.7\%) were found to have clinically significant PE. Increase in the Haller Index was correlated with higher MHD $(p<0.001)$. Through the use of individually optimized patient positioning and respiratory gating, MHD was not statistically different in patients with $P E$ compared to those without ( $p=0.093$ ). Conclusion: Although PE deformity is associated with unfavorable geometry in patients undergoing left-sided breast irradiation, carefully selected treatment technique can maintain acceptable MHD.
\end{abstract}

Adjuvant radiation therapy for women with breast cancer is associated with a reduction in local recurrence and, in many patients, a survival benefit $(1,2)$. As risk of cardiac events is correlated with the mean heart radiation dose (MHD), radiation oncological planning often focuses on techniques to reduce the MHD in women with left-sided breast cancer (3). Individual anatomic variation, including breast size, breast ptosis, tumor bed location, heart position and chest wall shape, all impact the ability of the radiation oncologist to achieve optimal MHD. In particular, pectus excavatum (PE) has been identified as a challenging situation for treatment planning in breast cancer.

This article is freely accessible online.

Correspondence to: Suzanne B. Evans, MD, MPH, 35 Park Street, LL509, New Haven, CT 06519, U.S.A. Tel: +1 2032002100, Fax:

+12037854622, e-mail: suzanne.evans@yale.edu

Key Words: Breast radiotherapy, mean heart dose, pectus excavatum.
PE is a congenital deformity of the chest wall associated with sternal depression. It accounts for about $90 \%$ of anterior chest wall disorders and has an incidence of one in 400 to one in 1,000 births (4). PE is about four times more prevalent in males than females (5). Classically, PE has been measured on computed tomography (CT) by the Haller index (HI), which is used to determine which patients might benefit from surgical correction. PE is well recognized as a condition which may increase MHD during left-sided breast radiation, prompting the investigation of several radiotherapy techniques to address this clinical problem, including intensity-modulated radiation therapy (6), deep inspiration breath-hold technique (DIBH) (7), and lateral decubitus positioning (8).

The purpose of this analysis was to determine the incidence of PE in women treated with radiation therapy for breast cancer at our Institution and to define anatomical chest wall metrics predictive of elevated MHD in patients undergoing breast radiotherapy.

\section{Patients and Methods}

Data source and study cohort. We retrospectively reviewed the records of 273 patients consecutively simulated for left-sided breast cancer or at our Institution between January 2013 and December 2014. The Yale Institutional Review Board approved this study (\#150301553). Patients underwent 3-dimensionsal conformal planning, with either forward planned fixed gantry field-in-field technique or wedges as indicated by individual patient geometry. Cardiac segmentation included both cardiac muscle and the pericardium, starting at the branch point of the pulmonary arteries and continuing inferiorly to the cardiac apex. The MHD was recorded from the treatment planning system using heterogeneity corrections (Eclipse Version 13.6; Varian, Palo Alto, CA, USA). Contribution to MHD from supraclavicular, axillary, or boost fields was not considered in the measurement of MHD. Patients with PE who were treated with DIBH were also retrospectively re-planned on their free breathing (FB) CT scan for comparison.

Variables. Patients were classified as having PE based on physical examination at the time of consult, which was confirmed upon review of axial imaging (Figure 1). Thoracic surgeons initially developed the HI to quantify the degree of the pectus deformity by 


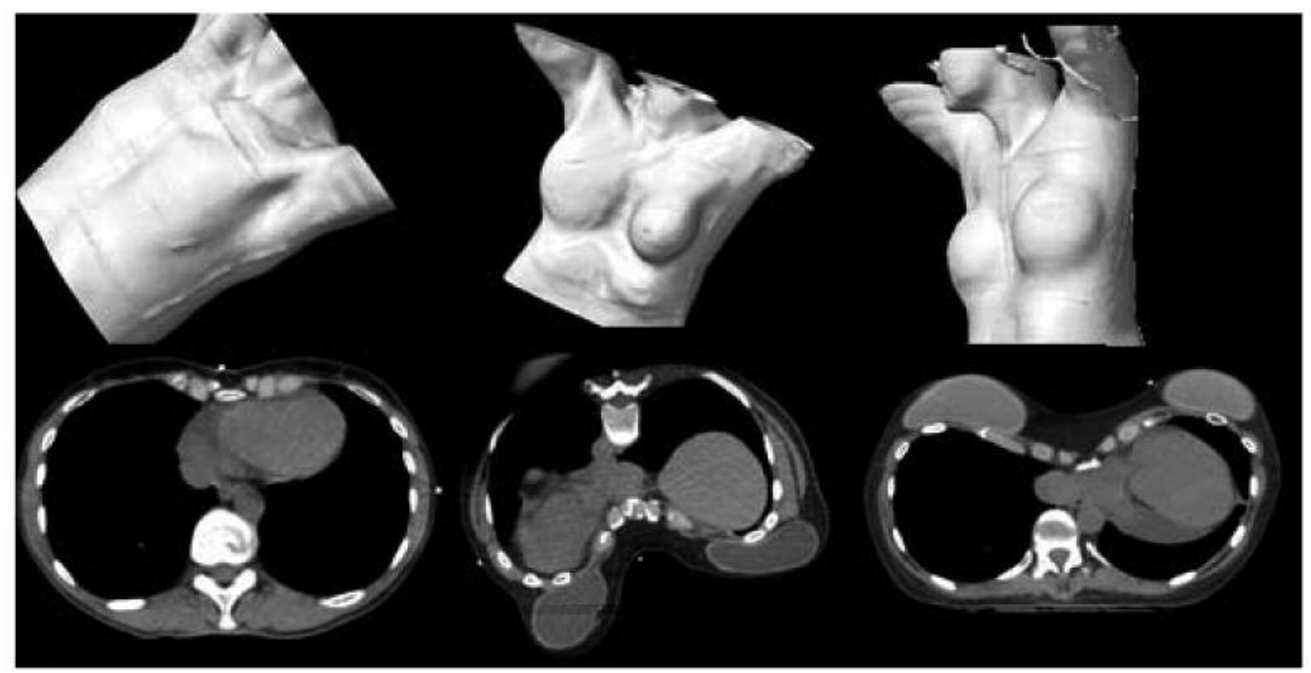

Figure 1. Example of patients with clinically apparent pectus excavatum. Skin rendering (top) and corresponding axial imaging (bottom) for three patients with clinically apparent pectus excavatum. Note the prominent sternal depression visible on external examination.

comparing the ratio of the lateral diameter of the chest and the distance from sternum to anterior vertebral body at the point of maximal depression (Figure 2) on CT scan (9). Among thoracic surgeons, a calculated HI of $>3.25$ designates PE which may benefit from surgical correction. For this analysis, HI was calculated on the FB scan. As the HI was designed to identify women with PE in need of surgical correction, and not to define significant PE for radiation oncologists (Figure 3), the depth of sternal depression (DSD) was developed by our group. The DSD was measured at the same axial location as the $\mathrm{HI}$ and quantifies the concavity of the sternal deformity (Figure 4).

Other variables of interest included age, cancer histology (invasive $v s$. ductal carcinoma in situ), surgical procedure (lumpectomy vs. mastectomy), receipt of chemotherapy, treatment volume (whole vs. partial breast), patient positioning (supine vs. prone), prescription dose, fraction size, respiratory gating, and total heart volume.

Statistical analysis. Chi-square tests and independent sample t-tests were used to compare characteristics between patients with PE and those without for categorical and continuous variables, respectively. Variables that were associated $(p<0.10)$ with MHD on bivariate linear regression were used to create multivariable linear regression models to predict MHD by forced entry. For continuous variables, residuals were plotted and found to follow a normal distribution. A two-sided alpha value of 0.05 was used to define statistical significance for all analyses. Analyses were performed using IBM SPSS statistics version 22 (SPSS, Armonk, NY, USA).

\section{Results}

Patient characteristics. The median age was 58 years (range $=27-84$ years) and most patients had invasive breast cancer $(81.1 \%)$. Most patients $(82.7 \%)$ underwent lumpectomy, $38.5 \%$ received chemotherapy, and $2.9 \%$

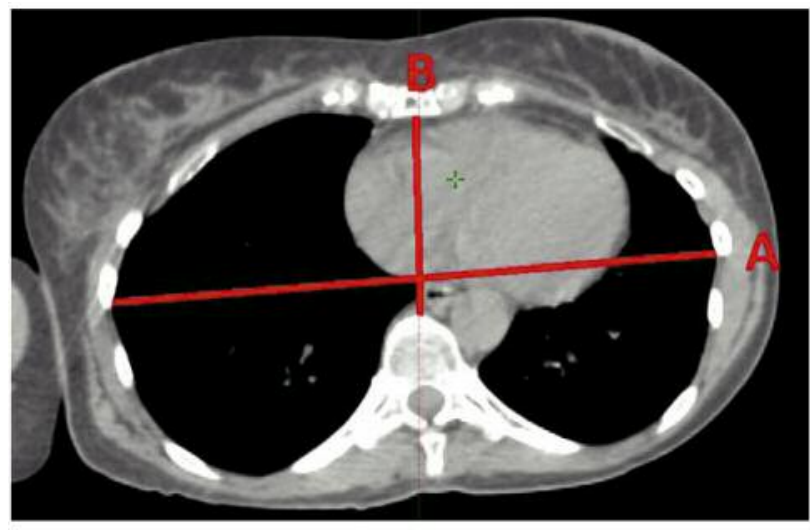

Figure 2. Haller index calculation. The Haller index was determined by comparing the ratio of the lateral diameter of the chest within the rib cage (' $A$ ') to the distance between the sternum and vertebral body (' $B$ ') at the point of maximal depression. To find the axial slice showing the maximal point of depression, the sagittal reconstruction was viewed and the most distal point on the sternum which was closest to the anteriormost point on the vertebral body corresponding to that slice was selected. Distance 'A' was measured from the most medial aspect of the rib or intercostal muscles on that slice to the opposite side at the mid axillary line. Distance ' $B$ ' was measured from the posterior aspect of the sternum on that slice to the anterior-most point on the vertebral body. The HI was then calculated by dividing ' $A$ ' by ' $B$.'

received partial breast radiation. The median MHD for the entire cohort was 172 cGy (interquartile range=126-267 cGy) and $10(3.7 \%)$ patients were found to have clinically apparent PE on clinical examination which was confirmed 


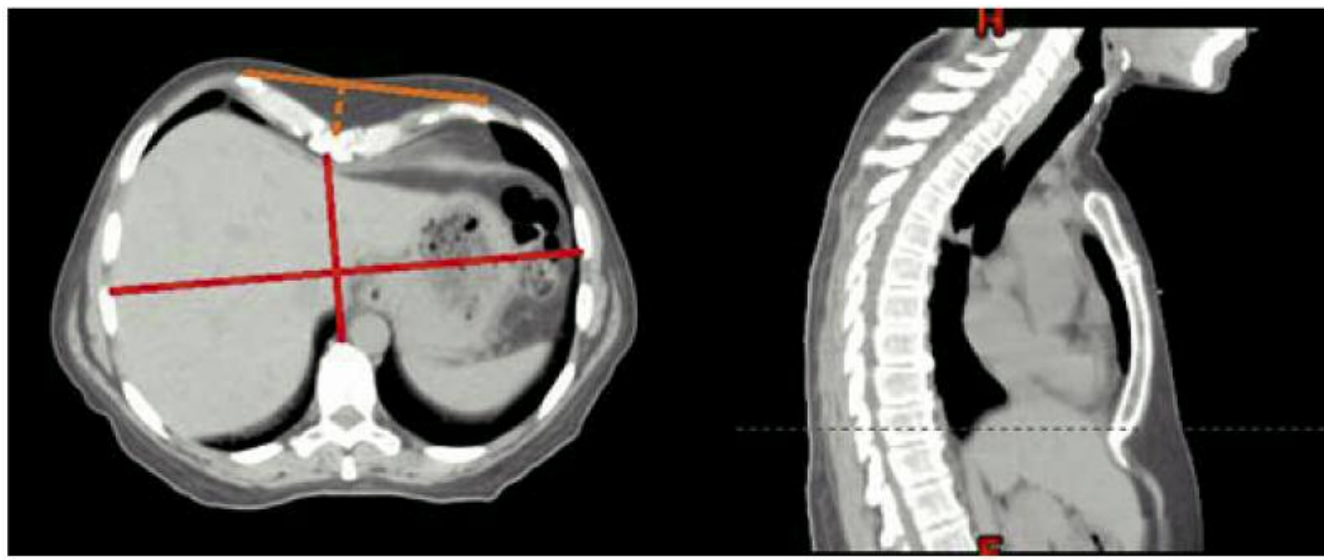

Figure 3. Failure of Haller index (2.51 in this instance) to identify clinically identifiable pectus excavatum, while the depth of sternal depression was $2.7 \mathrm{~cm}$. However, note that in this patient, the predominant chest wall shape is convex on the sagittal image, which may also explain why depth of sternal depression failed to be a good predictor of mean heart dose.

on review of CT imaging. Unadjusted analysis revealed that patients with PE were significantly younger than those without $(p<0.001)$ and had greater DSD $(p<0.001)$ (Table I). The HI trended towards significance as a predictor of clinically identifiable PE $(p=0.052)$, as defined above.

Chest wall deformity and MHD. For patients with PE, a variety of strategies were employed to optimize their treatment (Table II). As a result of these techniques, patients with PE had a MHD that was not significantly different from that seen in patients without PE (297 cGy $v s .216 \mathrm{cGy}, p=0.093)$. Of note, one patient simulated with PE did not undergo radiotherapy due to a planned MHD of 1,269 cGy and marginal indications for post-mastectomy radiation therapy. The average MHD for the three patients with PE treated with DIBH was $196 \mathrm{cGy}$. For these same patients re-planned using supine FB technique, the average MHD was 438 cGy, which was not a statistically significant difference in this small cohort $(p=0.055)$ by independent sample $t$-test. A reduction in MHD was seen in all three patients $(70.1 \%, 54.9 \%$, and $30.5 \%$ reduction in MHD) using DIBH.

Anatomical predictors of MHD in the entire cohort. Unadjusted and adjusted predictors of MHD are shown in Table III. Since only the HI was predictive of MHD $(p<0.001)$ among anatomical parameters using bivariate linear regression, a multivariable linear regression was created for the HI. A significant linear regression equation was derived to model MHD by $\mathrm{HI}$ adjusting for other significant variables, with an $\mathrm{R}^{2}$ of 0.310 . A rise in $\mathrm{HI}$ of 1 was associated with an increased MHD of $75 \mathrm{cGy}$ $(p<0.001)$.

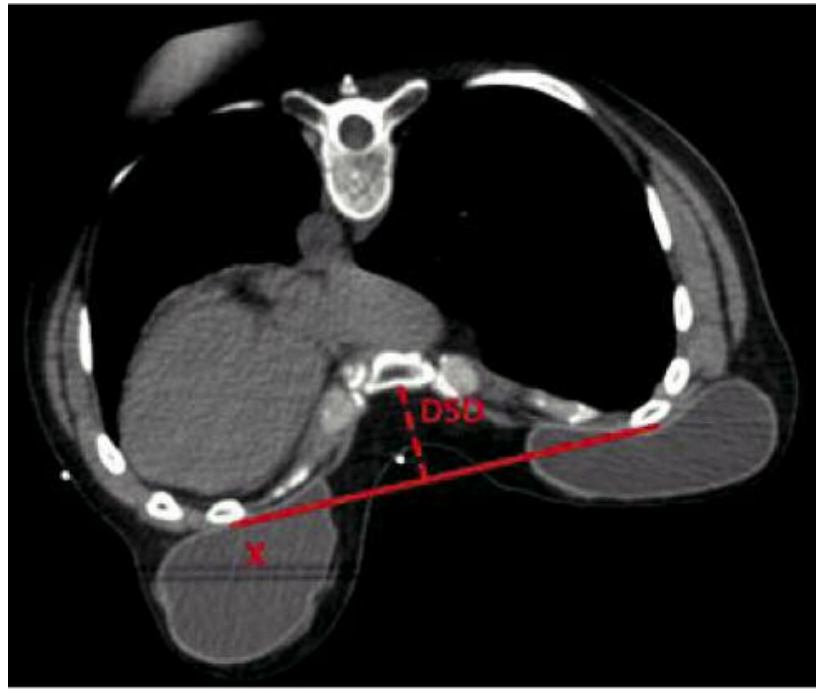

Figure 4. Calculation of the depth of sternal depression (DSD). The maximal depth of sternal depression was measured on the same computed tomographic scan slice as that from which the Haller index was derived (point of maximal chest wall deformity). A line was drawn parallel to the chest wall at the anterior-most point of the ribs (solid line). The maximal DSD was measured from this line to the surface of the sternum (dashed line). In this patient, the DSD measured $3.8 \mathrm{~cm}$.

\section{Discussion}

Our single-institution, retrospective analysis of left-sided breast cancer radiotherapy showed that $3.7 \%$ of patients had clinically identifiable $\mathrm{PE}$. As a result of techniques specifically intended to reduce MHD, our PE cohort did not have a significantly higher MHD than patients without PE. 
Table I. Patient characteristics by chest wall deformity status.

\begin{tabular}{|c|c|c|c|}
\hline & Pectus excavatum $(n=10)$ & No pectus excavatum $(n=263)$ & Unadjusted $p$-value \\
\hline Mean age at consult (years) & 51.5 & 58.4 & $<0.001$ \\
\hline Breast cancer histology & & & 0.38 \\
\hline Invasive & $6(66.7 \%)$ & $213(81.6 \%)$ & \\
\hline DCIS & $3(33.3 \%)$ & $48(18.4 \%)$ & \\
\hline Surgery type & & & 0.69 \\
\hline Lumpectomy & $8(80.0 \%)$ & $217(82.8 \%)$ & \\
\hline Mastectomy & $2(20.0 \%)$ & $45(17.2 \%)$ & \\
\hline Chemotherapy & & & 0.33 \\
\hline No & $8(80.0 \%)$ & $160(60.8 \%)$ & \\
\hline Yes & $2(20.0 \%)$ & $103(39.2 \%)$ & \\
\hline Treatment volume & & & 0.26 \\
\hline Whole breast irradiation & $9(90.0 \%)$ & $256(97.3 \%)$ & \\
\hline Partial breast irradiation & $1(10.0 \%)$ & $7(2.7 \%)$ & \\
\hline Patient setup & & & 1.00 \\
\hline Prone & $2(20.0 \%)$ & $56(21.3 \%)$ & \\
\hline Supine & $8(80.0 \%)$ & $207(78.7 \%)$ & \\
\hline Deep inspiratory breath-hold planned & & & 0.48 \\
\hline No & $6(60.0 \%)$ & $118(71.8 \%)$ & \\
\hline Yes & $4(40.0 \%)$ & $74(28.2 \%)$ & \\
\hline Average, mean heart dose (cGy) & 297 & 216 & 0.093 \\
\hline Mean heart volume (cc) & 482 & 485 & 0.94 \\
\hline Mean Haller Index & 3.54 & 2.33 & 0.052 \\
\hline Mean depth of sternal depression $(\mathrm{cm})$ & 1.78 & 0.012 & $<0.001$ \\
\hline Mean prescription dose (cGy) & 4686 & 4753 & 0.63 \\
\hline Mean daily fraction size & 232 & 220 & 0.64 \\
\hline 200cGy & 7 & 193 & \\
\hline $265 \mathrm{cGy}$ & 0 & 14 & \\
\hline $267 \mathrm{cGy}$ & 2 & 45 & \\
\hline $385 \mathrm{cGy}$ & 1 & 7 & \\
\hline 180 or $250 \mathrm{cGy}$ & 0 & 4 & \\
\hline
\end{tabular}

DCIS: Ductal carcinoma in situ.

For our patients with $\mathrm{PE}$ who received treatment via the DIBH technique, MHD was reduced by an average of 242 cGy (or 55\%) but this was only a strongly nonsignificant trend $(p=0.055)$ due to the small patient numbers. DIBH has been used to reduce radiation exposure to the heart in patients undergoing left-sided whole-breast radiotherapy (10-13). In patients with $\mathrm{PE}$, there are fewer data suggesting its efficacy. For this limited sample of patients, a consistent reduction in MHD of variable magnitude (from $30 \%$ to $70 \%$ ) was demonstrated.

When unfavorable chest wall anatomy has been identified, modifications to standard tangential irradiation have been suggested. In order to solve this difficult clinical problem, the use of helical tomotherapy (6) or volumetric-modulated arc therapy (14), intensity-modulated radiation, and isocentric lateral decubitus technique (8) have been proposed. Our data show that excellent MHD can be obtained without resorting to resource-intensive techniques such as helical tomotherapy or intensity-modulated radiation. The reproducibility of this finding in a wider population would require further study.
Table II. Average mean heart dose (MHD) by chest wall deformity status and treatment technique.

N Average MHD (cGy)

\begin{tabular}{lcc}
\hline PE with supine DIBH & 3 & 196 \\
PE with prone FB & 2 & 167 \\
PE with supine FB & 3 & 249 \\
PE with partial breast irradiation & 1 & 34.7 \\
Non-PE with supine DIBH & 74 & 163 \\
Non-PE with prone FB & 56 & 165 \\
Non-PE with supine FB & 125 & 276.9 \\
Non-PE with partial breast irradiation & 7 & 59.8 \\
\hline
\end{tabular}

PE: Pectus excavatum; DIBH: deep inspiratory breath hold, FB: free breathing.

In this study, the novel parameter DSD was significantly associated with clinically identifiable PE. However, in the full cohort, DSD was not significantly associated with MHD. As shown in Figure 3, clinical identification of PE 
Table III. Predictors of mean heart dose using linear regression ( $n=273)$.

\begin{tabular}{lccc}
\hline & $\begin{array}{c}\text { Univariate } \\
p \text {-value }\end{array}$ & $\begin{array}{c}\text { Standardized } \\
\text { beta coefficient }\end{array}$ & $\begin{array}{c}\text { Adjusted } \\
p \text {-value }\end{array}$ \\
\hline Haller index & $<0.001$ & 0.254 & Lower Haller index associated with reduced MHD \\
Depth of sternal depression $(\mathrm{cm})$ & 0.897 & 0.008 & $<0.001$ \\
Age at consult (years) & 0.196 & -0.079 & - \\
DCIS $v$. invasive & 0.281 & -0.066 & - \\
Lumpectomy $v s$. mastectomy & $<0.001$ & -0.266 & Lumpectomy associated with reduced MHD \\
Chemotherapy $v s$. no chemotherapy & $<0.001$ & 0.254 & No chemotherapy associated with reduced MHD \\
Whole $v s$. partial breast irradiation & 0.002 & 0.188 & Partial breast irradiation associated with reduced MHD \\
DIBH $v s$. FB & 0.006 & -0.168 & 0.058 \\
Heart volume (cc) & 0.051 & 0.118 & DIBH associated with reduced MHD \\
Prescription dose (cGy) & $<0.001$ & 0.369 & Lower prescription dose associated with reduced MHD \\
\hline
\end{tabular}

DCIS: Ductal carcinoma in situ; DIBH: deep inspiratory breath-hold technique; FB: free breathing technique.

does not necessarily indicate that a patient will have the classic altered rotation of the cardiac silhouette seen in patients with severe PE. As experienced breast radiation oncologists, the clinical examination finding of a depressed sternum is notable due to the potential problems with treatment planning which may ensue from this anatomic variant. However, the magnitude of this depression and its impact on internal cardiac anatomy cannot be discerned from physical examination. As such, DSD did not reliably predict for elevated MHD. Several studies have defined quantitative metrics based on planning CT scans to identify patients with unfavorable cardiac anatomy. For example, highly unfavorable cardiac anatomy has been defined by measuring the cardiac contact distance in both the axial and para-sagittal planes (15). Another study did not find the HI to be predictive of MHD, but did find that the number of consecutive $2 \mathrm{~mm}$ axial FB CT slices where the heart contacted the anterior chest wall was most predictive of MHD and the need for breath-hold technique (7). Our study did find the HI to be significantly associated with MHD after adjusting for prescription dose, heart volume, and treatment technique. Although the HI was not specifically used to identify candidates for cardiac avoidance techniques in our cohort, this practice may be useful in radiotherapy for left-sided cases. This study is strengthened by a fairly large sample size for a retrospective study, and the inclusion of all patients sequentially simulated, reducing the likelihood of sampling bias.

We acknowledge that the $\mathrm{HI}$ is one of several potential anatomic metrics that predict MHD. In our series, the correlation between $\mathrm{HI}$ and MHD was statistically significant, but not fully informative on its own $\left(\mathrm{R}^{2}=0.31\right)$. For any patient, there are many considerations that impact the degree of cardiac exposure to radiation: tumor bed location, breast ptosis, clinical stage, and values of the patient as well as the treating clinician (i.e. risk-benefit assessment of cardiac shielding). The incidence of PE determined in our institution does appear higher $(3.7 \%)$ than what has been reported in larger studies, which increases the likelihood of over-detection attributable to an imprecise clinical definition. Regardless of these subtleties, our low overall MHD which was also replicated in the PE population indicates that cardiac avoidance can be obtained without use of resource-intensive techniques.

Although PE deformity is associated with unfavorable geometry in patients undergoing left-sided breast irradiation, carefully selected treatment technique can maintain acceptable MHD. The HI can be useful in identifying women likely to benefit from cardiac avoidance techniques. DIBH technique should be considered to reduce cardiac radiation dose in patients with PE.

\section{Conflicts of Interest}

John M. Stahl, MD, Julian C. Hong, MD, MS, Lynn D. Wilson, MD, MPH, Susan A. Higgins, MD, and Benjamin H. Kann, MD have none. Suzanne B. Evans, MD, MPH has an institutional research funding disclosure for 21st Century Oncology. Nataniel Lester-Coll, MD received an honorarium from Elekta $\mathrm{AB}$, unrelated to the current work.

\section{References}

1 Early Breast Cancer Trialists' Collaborative Group, Darby S, McGale P, Correa C, Taylor C, Arriagada R, Clarke M, Cutter D, Davies C, Ewertz M, Godwin J, Gray R, Pierce L, Whelan T, Wang $\mathrm{Y}$ and Peto R: Effect of radiotherapy after breastconserving surgery on 10-year recurrence and 15-year breast cancer death: meta-analysis of individual patient data for 10,801 women in 17 randomised trials. Lancet 378: 1707-1716, 2011.

2 Early Breast Cancer Trialists' Collaborative Group, McGale P, Taylor C, Correa C, Cutter D, Duane F, Ewertz M, Gray R, 
Mannu G, Peto R, Whelan T, Wang Y, Wang Z and Darby S Effect of radiotherapy after mastectomy and axillary surgery on 10-year recurrence and 20-year breast cancer mortality: metaanalysis of individual patient data for 8135 women in 22 randomised trials. Lancet 383: 2127-2135, 2014.

3 Darby SC, Ewertz M, McGale P, Bennet AM, Blom-Goldman U, Bronnum D, Correa C, Cutter D, Gagliardi G, Gigante B, Jensen MB, Nisbet A, Peto R, Rahimi K, Taylor C and Hall P: Risk of ischemic heart disease in women after radiotherapy for breast cancer. N Engl J Med 368: 987-998, 2013.

4 Fokin AA, Steuerwald NM, Ahrens WA and Allen KE: Anatomical, histologic, and genetic characteristics of congenital chest wall deformities. Semin Thorac Cardiovasc Surg 21: 4457, 2009.

5 Fonkalsrud EW: 912 open pectus excavatum repairs: changing trends, lessons learned: one surgeon's experience. World J Surg 33: 180-190, 2009.

6 Uhl M, Sterzing F, Habl G, Schubert K, Holger H, Debus J and Herfarth K: Breast cancer and funnel chest. Comparing helical tomotherapy and three-dimensional conformal radiotherapy with regard to the shape of pectus excavatum. Strahlenther Onkol 188: 127-135, 2012.

7 Lee G, Rosewall T, Fyles A, Harnett $\mathrm{N}$ and Dinniwell RE: Anatomic features of interest in women at risk of cardiac exposure from whole breast radiotherapy. Radiother Oncol 115: 355-360, 2015.

8 Bollet MA, Campana F, Kirova YM, Dendale R, Saliou MG, Rosenwald JC and Fourquet A: Breast radiotherapy in women with pectus excavatum (funnel chest): is the lateral decubitus technique an answer? A dosimetric study. Br J Radiol 79: 785790, 2006

9 Haller JA, Jr., Kramer SS and Lietman SA: Use of CT scans in selection of patients for pectus excavatum surgery: a preliminary report. J Pediatr Surg 22: 904-906, 1987.
10 Remouchamps VM, Letts N, Vicini FA, Sharpe MB, Kestin LL, Chen PY, Martinez AA and Wong JW: Initial clinical experience with moderate deep-inspiration breath hold using an active breathing control device in the treatment of patients with leftsided breast cancer using external beam radiation therapy. Int $\mathrm{J}$ Radiat Oncol Biol Phys 56: 704-715, 2003.

11 Wang W, Purdie TG, Rahman M, Marshall A, Liu FF and Fyles A: Rapid automated treatment planning process to select breast cancer patients for active breathing control to achieve cardiac dose reduction. Int J Radiat Oncol Biol Phys 82: 386-393, 2012.

12 Hayden AJ, Rains M and Tiver K: Deep inspiration breath hold technique reduces heart dose from radiotherapy for left-sided breast cancer. J Med Imaging Radiat Oncol 56: 464-472, 2012.

13 Swanson T, Grills IS, Ye H, Entwistle A, Teahan M, Letts N, Yan D, Duquette J and Vicini FA: Six-year experience routinely using moderate deep inspiration breath-hold for the reduction of cardiac dose in left-sided breast irradiation for patients with early-stage or locally advanced breast cancer. Am J Clin Oncol 36: 24-30, 2013.

14 Haertl PM, Pohl F, Weidner K, Groeger C, Koelbl O and Dobler $\mathrm{B}$ : Treatment of left sided breast cancer for a patient with funnel chest: volumetric-modulated arc therapy $v s$. 3D-CRT and intensity-modulated radiotherapy. Med Dosim 38: 1-4, 2013.

15 Hiatt JR, Evans SB, Price LL, Cardarelli GA, Dipetrillo TA and Wazer DE: Dose-modeling study to compare external beam techniques from protocol NSABP B-39/RTOG 0413 for patients with highly unfavorable cardiac anatomy. Int $\mathrm{J}$ Radiat Oncol Biol Phys 65: 1368-1374, 2006.

Received August 17, 2016

Revised September 2, 2016

Accepted September 12, 2016 